\title{
Research on Fairing design and CFD Analysis of Submarine Pipeline Inspection ARV
}

\author{
Xiaojian Jin ${ }^{1}$, Shunqi Yang ${ }^{2}$, Hai Chen ${ }^{1}$ \\ ${ }^{1}$ Beijing University Of Posts And Telecommunications, Beijing, China \\ ${ }^{2}$ Kunming Shipborne Equipment Research \& Test Center, Kunming, China
}

\begin{abstract}
Along with the fast development of the ocean exploitation, the cost-effective requirement of autonomous \& remotely operated vehicle (ARV), which can perform more complicated missions such as the oil exploitation and the inspection of the submarine pipeline is more urgent. The submarine pipeline inspection ARV can help us better understand, protect and efficiently utilize them for human welfare. Fairing design of a new detection ARV are introduced in this paper. In order to select an appropriate thruster that will achieve the required speed of the ARV, the ANSYS-CFX tools are used to predicted the drag force. The CFD results reveal the distribution of velocity and pressure values of the ARV. In order to verify the CFD modeling process, a towed body was developed and analyzed, compared against the corresponding physical test data.
\end{abstract}

\section{Introduction}

Covering about two-thirds of the earth, the ocean has a great eff ect on our lives on land. With the substantial consume of energy sources and increase of population, ocean research and exploitation gradually become the new development space of people surviving. Meanwhile, along with the development of offshore oil and gas in our country is more and more active, especially the deep oil and gas development, the offshore platform under service is increasing year by year. The submarine pipeline is also increasing year by year as an important part of the offshore platform in development of sea oil. The environment of the submarine pipeline is strong tidal zone gulf of high risk, urgent tide, tidal range; the transmission medium is a high-sulphur crude oil. In such a complex and harsh service environment, safe and stable operation of the submarine pipeline is the most important issue to consider. Any failure of these submarine pipelines will not only affect productivity negatively but also cause tremendous environmental hazards. According to the present international and domestic requirement of the regulations for the underwater pipeline, routine or annual inspection to the pipelines should be taken to ensuring the safely or longer period of using of the pipeline.

In recent years, unmanned underwater vehicles (UUVs) have demonstrated their capabilities in many important applications in fields such as oceanographic research, offshore oil and gas industry, and military operations. Thus, as the necessary tool for submarine pipeline detection, the underwater robot submarine pipeline detection system is playing an increasingly important value. Usually, submarine pipeline inspections are performed by remote operated vehicle (ROV) driven by human operators on water surface. However, this task is often challenging, especially in conditions of poor visibility or in presence of strong currents; and ROV's working need support vessel support, low efficiency, high cost. Along with the fast development of the ocean exploitation, the cost-effective requirement of autonomous \& remotely operated vehicle (ARV), which can perform more complicated missions such as the oil exploitation and the inspection of the submarine pipeline, is more urgent [1-3]. ARV has received much attention since they can be an effective tool to explore the ocean and efficiently utilize the ocean resources. ARV been used for various applications including military operations, environmental systems, scientific missions, ocean mining, and gas and oil industry. With the demand of ocean engineering and offshore petroleum exploitation, ARV which represents the direction of underwater vehicle has gained rapid development. As a sea power multiplier, ARV can not only be used in various important military applications, but is an important tool for peaceful exploitation of the ocean. ARV can carry the temperature, salinity, sonar detection instrument for collecting marine environment data and topography data, in order to provide information to the exploration of seabed resources, as well as in offshore drilling, submarine pipeline laying and maintenance of submarine pipeline. The ARV for Submarine Pipeline Inspection mission should exhibit compact size, which allows for economical overnight shipping, less manned deployment, and operation capability, together with eliminated need for large dedicated transporting vessels and costly special 
handling equipment. So ARV has been widely applied as an important component of ocean high-tech. The submarine pipeline inspection ARV cruises in the fixed date, which can independently find and track pipeline on the seabed. It not only can observe the state of pipeline and the circumstance near the pipeline, but also can warn the coming fault. Thus, the most effective way to ensure safe and efficient operation for the long-distance submarine pipeline is to perform periodic inspects for them by ARV. [4]

The submarine pipeline inspection ARV for oil industry has been developed and its design evaluated by CFD an analysis such as a resistance test. The development of commercial codes for CFD analysis now make it possible to predict drag and propulsion performance of an underwater robot without using a physical model test basin [5]. There are many efforts at predicting drag forces of underwater vehicles by CFD analysis and validating CFD simulation methods in design processes [6-7]. CFD simulations are also compared with in-service data for the self-propelled performance of an Autonomous Underwater Vehicle [8]. The present paper focuses on design issues with fairing of the ARV. A CFD analysis was first used to conduct the resistance test necessary to predict the total drag force for selecting an appropriate thruster that will achieve the required speed of the $A R V$. In order to verify the CFD modelling process, a model of a towed body was developed and analysed and compared against the corresponding physical test data.

The rest of this paper is organized as follows. Section 2 provides the mechanical design for submarine pipeline inspection ARV with 300 meters depth. The fairing design for ARV in Section 3. The CFD Setup for Predicting the ARV drag force is presented in Section 4. Section 5 presents some CFD Analysis results. Some conclusions are given in Section6.

\section{Mechanical design for submarine pipeline inspection ARV}

Mechanical systems that have excellent environmental resistance are needed. An underwater robot's mechanical structure can be divided into three functional subsystems: frame, pressure vessel, and fairing. A fairing that covers all or parts of an underwater robot offers some advantages, such as an aesthetically pleasing exterior, a decreased drag force, and protection. A fairing is desirable for ARV, due to limited on-board power sources for required hydrodynamic performance. The ARV, shown in Figure 1, was designed as a flat shape with hydrodynamic contour to keep stable and reduce the drag force on the body in the horizontal direction and designed for a maximum operating depth of up to $300 \mathrm{~m}$, is composed of an titanium alloy frame that supports two cylinder-shaped hulls of $0.3 \mathrm{~m}$ in diameter and engineering plastic fairing skin in the outside cover. This design offers a good hydrodynamic performance and a large space for housing the equipment while maintaining a compact size that allows us to operate the vehicle from conventional ships.

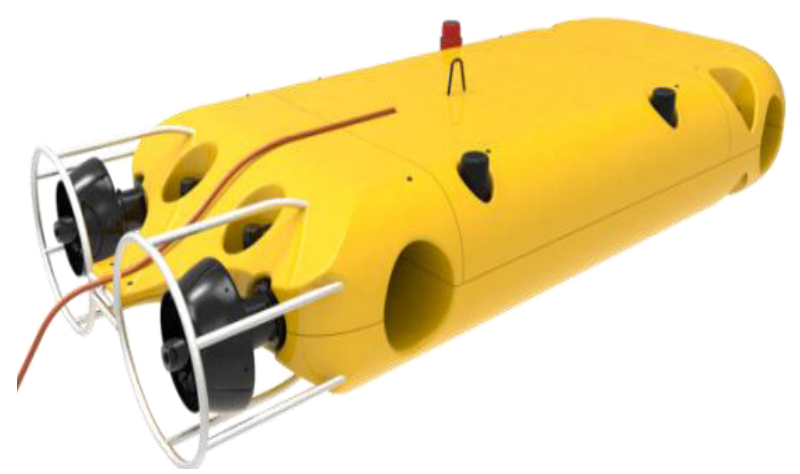

Figure 1. Assembly of the ARV

The ARV measures $2.8 \mathrm{~m}$ in length, $1.3 \mathrm{~m}$ width and $0.42 \mathrm{~m}$ in height. The design speed for the initial design of the ARV is $2 \mathrm{knots}$ and the maximum speed is $6 \mathrm{knot}$.

The ARV frame is made of TiAl6v4 titanium alloy and is composed of one I-shaped pillar screwed to three U-shaped profiles that serve as backbone to each one of the three cylinder-shaped hulls. The ARV pressure vessel is made of hard anodized 6082-T6 aluminium alloy and contains two cylindrical pressure housings which contain mainly the control electronics and battery cluster. The ARV flotation modules in between skin and pressure vessel, made of an epoxy composite foam with a density of $270 \mathrm{~kg} / \mathrm{m}^{3}$, are placed on the top part of the vehicle. Their principal mission is to make the vehicle almost neutrally buoyant.

\section{Fairing design}

The frame, pressure vessel and flotation modules that compose the ARV vehicle are covered with a thermoformed ABS plastic skin whose streamlined shape is based on the Myring hull profile equations [9]. The ARV fairing skin provides protection to the sensible equipment and reduces the drag of the vehicle. The flow curve can be formulated as follows:

$$
y=(x-f) \cdot \sqrt{L_{1} \cdot X-X^{2}} \cdot D /(2 \cdot k)
$$

Where, $D$ is height of ARV. And the flow calculation plot is given in Figure 2.

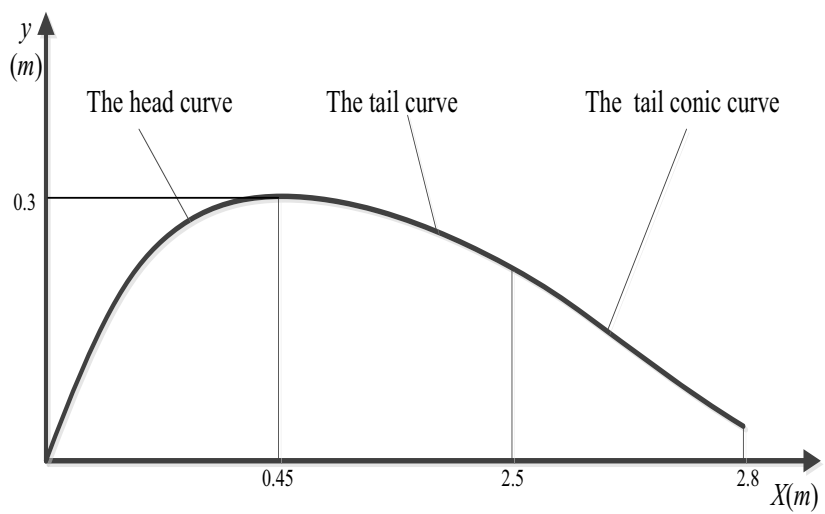

Figure2. Flow curve of the ARV 


\section{CFD Setup for Predicting the ARV drag force}

The fluid flow around the ARV has been modeled using the commercial CFD analysis code ANSYS-CFX 14.0. For these calculations, the fluid's motion is modeled using the incompressible, isothermal Reynolds-averagedNavier-Stokes (RANS) equations in order to determine the Cartesian flow field and pressure of the water around the ARV body. The equations consist of a general solution of the ensemble-averaged, steady-state, three dimensional Navier - Stokes equations, where the $\mathrm{k}-\varepsilon$ turbulence model ( $\mathrm{k}$ is turbulence kinetic energy and $\varepsilon$ is viscous dissipation rate) has been used to close the system of equations.

The continuity equation is given

$$
\frac{\partial \overline{u_{i}}}{\partial x_{i}}=0
$$

The $\mathrm{c}$ momentum equation is given

$$
\rho \frac{\partial \overline{u_{i}}}{\partial t}+\rho \overline{u_{j}} \frac{\partial \overline{u_{i}}}{\partial x_{j}}=\rho \bar{F}_{i}-\frac{\partial \bar{p}}{\partial x_{i}}+\frac{\partial}{\partial x_{j}}\left(\mu \frac{\partial \overline{u_{i}}}{\partial x_{j}}-\rho \overline{u_{i}^{\prime} u_{j}^{\prime}}\right)
$$

The intensity of turbulence $k$ equation is given

$$
\frac{\partial}{\partial t}(\rho k)+\frac{\partial}{\partial x_{i}}\left(\rho k u_{i}\right)=\frac{\partial}{\partial x_{j}}\left[\left(\mu+\frac{\mu_{t}}{\sigma_{k}}\right) \frac{\partial k}{\partial x_{j}}\right]+G_{k}-\rho \varepsilon
$$

The turbulent dissipation $\varepsilon$ equation is given $\frac{\partial}{\partial t}(\rho \varepsilon)+\frac{\partial}{\partial x_{i}}\left(\rho \varepsilon u_{i}\right)=\frac{\partial}{\partial x_{j}}\left[\left(\mu+\frac{\mu_{t}}{\sigma_{\varepsilon}}\right) \frac{\partial \varepsilon}{\partial x_{j}}\right]+G_{1 \varepsilon} \frac{\varepsilon}{k} G_{k}-G_{2 \varepsilon} \rho \frac{\varepsilon^{2}}{k}-R_{\varepsilon}$

(5)

where,

$$
\begin{gathered}
\mu_{t}=\rho C_{\mu} \frac{k^{2}}{\varepsilon} \\
G_{k}=-\rho \overline{u_{i}^{\prime} u^{\prime}} \frac{\partial \overline{u_{i}}}{\partial x_{j}} \\
R_{\varepsilon}=\frac{C_{\mu} \rho \eta^{3}\left(1-\eta / \eta_{0}\right)}{1+\beta \eta^{3}} \frac{\varepsilon^{2}}{k} \\
\eta=S k / \varepsilon
\end{gathered}
$$

and $\eta_{0}=4.48, \beta=0.013, C_{1 \varepsilon}=1.45, C_{2 \varepsilon}=0.0845$, $\sigma_{k}=1.0, \sigma_{\varepsilon}=1.3$.

The plane mesh of the domain is shown in Figure 3. The length of the domain is 7 times longer than the ARV body and width and height are 6 times longer. The length of the tank was made long enough to allow the wake from the ARV body to be observed and the width and height of the tank are also long enough to reduce the wall effect. The meshing operation was performed using ICEM CFD as shown in Figure 4. Layered (5 layers) meshes were used for generating the boundary layer elements around the ARV body while unstructured (Tetrahedral) meshes were employed for the region far from the body as they are not suitable to resolve the boundary layers adjacent to a solid body [10]. The specifications for the pre-processing stage in CFX as shown in Figure 5-10, including the mesh generation, are summarized in Table 1.

Table 1 Principal conditions employed in the CFD analysis

\begin{tabular}{|l|l|}
\hline Water tank size & $\mathbf{1 9 . 6} \mathbf{m} \times \mathbf{7 . 2} \times \mathbf{2 . 5 m}$ \\
\hline Turbulence model & $\mathrm{k}-\varepsilon$ (Scalable wall function) \\
\hline No. of the inflation layer & 5 \\
\hline Total no. of elements (nodes) & $320210(63144)$ \\
\hline No. of Tetrahedral elements & 320210 \\
\hline
\end{tabular}

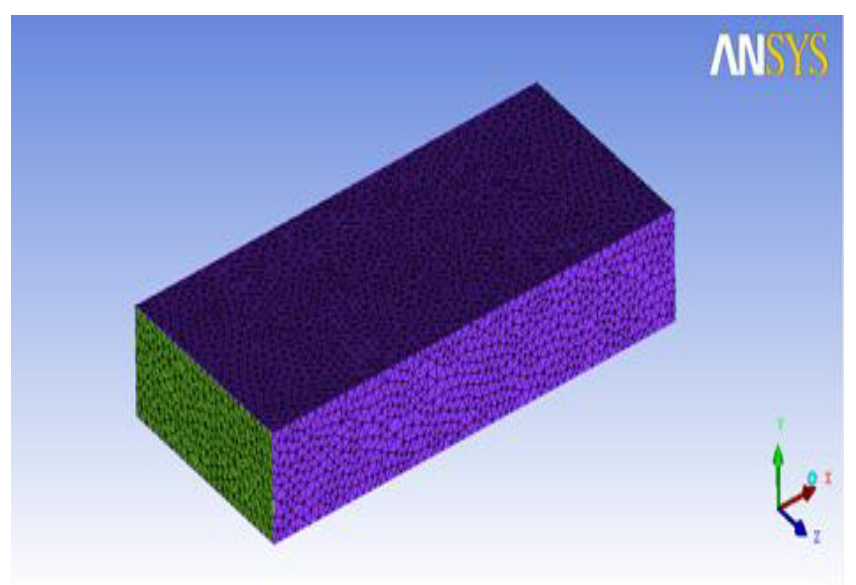

Figure 3. Review of the meshing results for ARV

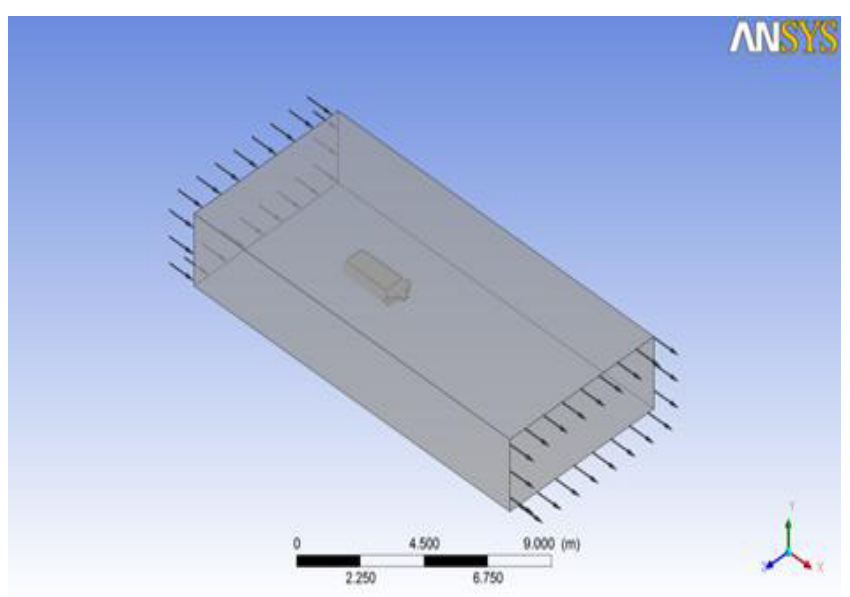

Figure 4. Pre-processing results for ARV

\section{CFD Analysis results}

A mesh convergence study was performed at the design speed ( $3 \mathrm{knots}$ ), and the results are displayed in Table 2. It was observed that once the number of cells for the case is reached the size of the reference, the variation in the total drag force (RT) was small considering the significant changes made to the simulation. The reference mesh size for the case was therefore considered to be of sufficient 
accuracy. The total drag force (RT) of the ARV body is obtained directly from the CFD analysis and shown in Table 3. Figure 5-10 shows velocity and pressure distribution at the lowest speed (1 knot), design speed (3knots) and highest speed (5knots) in this simulation test. In order to verify the CFD modeling method, a towed body was simulated and compared against the corresponding physical test data. It is found obviously that the calculation error between the simulation and the experimental results is less than $20 \%$. Thus the model is validated.

Table 2 Result of convergence study with ARV CFD model at the design speed

\begin{tabular}{|l|l|l|}
\hline Case & Number of cells Total & Drag Force( 3kn) \\
\hline Reference & 320210 & $114[\mathrm{~N}]$ \\
\hline $\begin{array}{l}\text { Reduced } \\
\text { mesh size-1 }\end{array}$ & 91852 & $232[\mathrm{~N}]$ \\
\hline $\begin{array}{l}\text { Reduced } \\
\text { mesh size-2 }\end{array}$ & 215585 & $129[\mathrm{~N}]$ \\
\hline $\begin{array}{l}\text { Increased } \\
\text { mesh size-1 }\end{array}$ & 1125787 & $88[\mathrm{~N}]$ \\
\hline
\end{tabular}

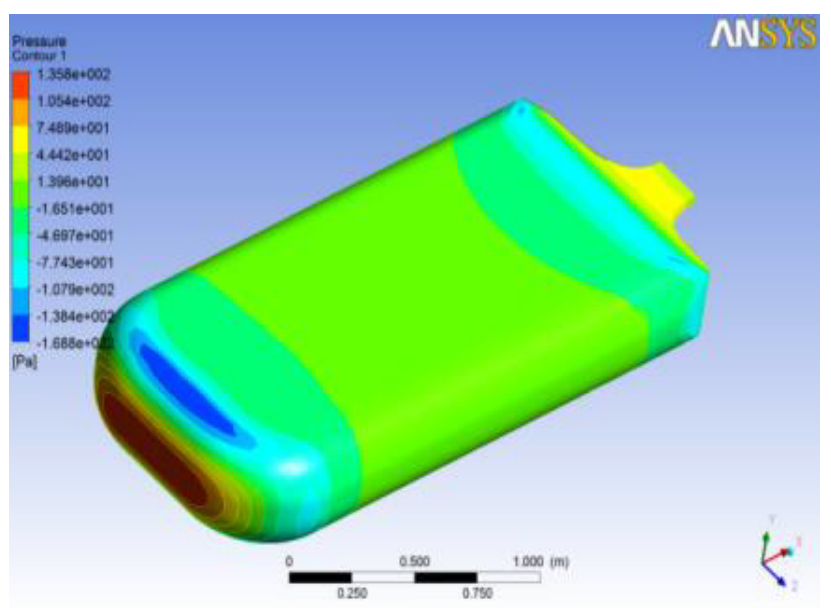

Figure 5. Velocity distribution at $1 \mathrm{kn}$

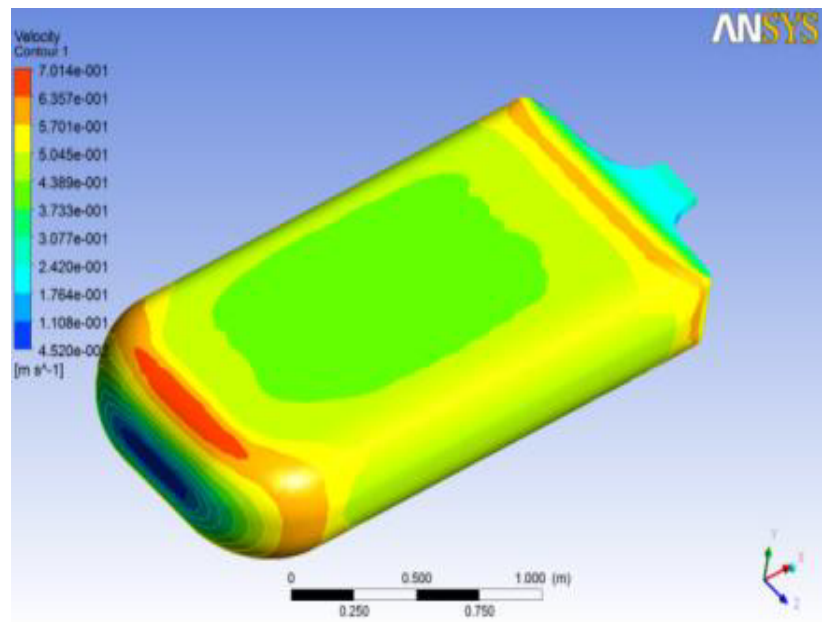

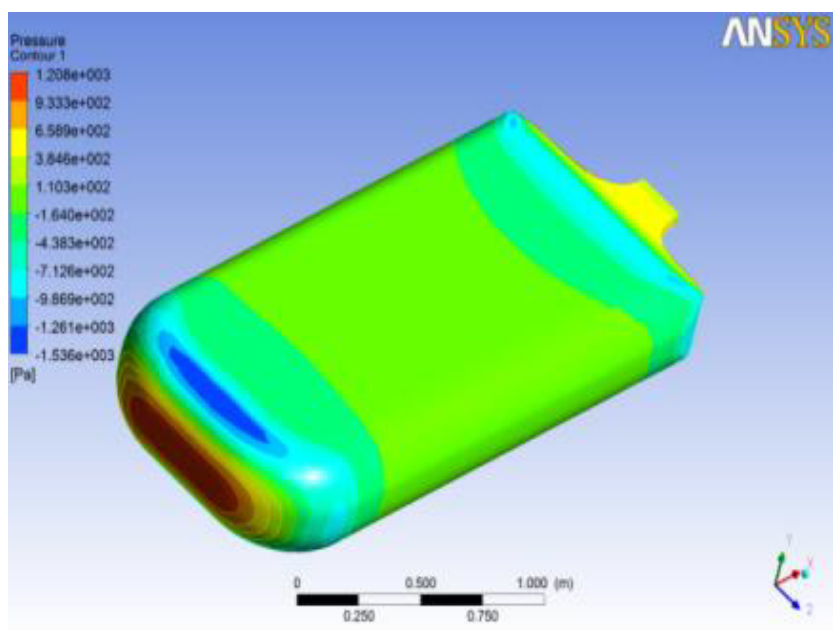

Figure 7. Velocity distribution at $3 \mathrm{kn}$

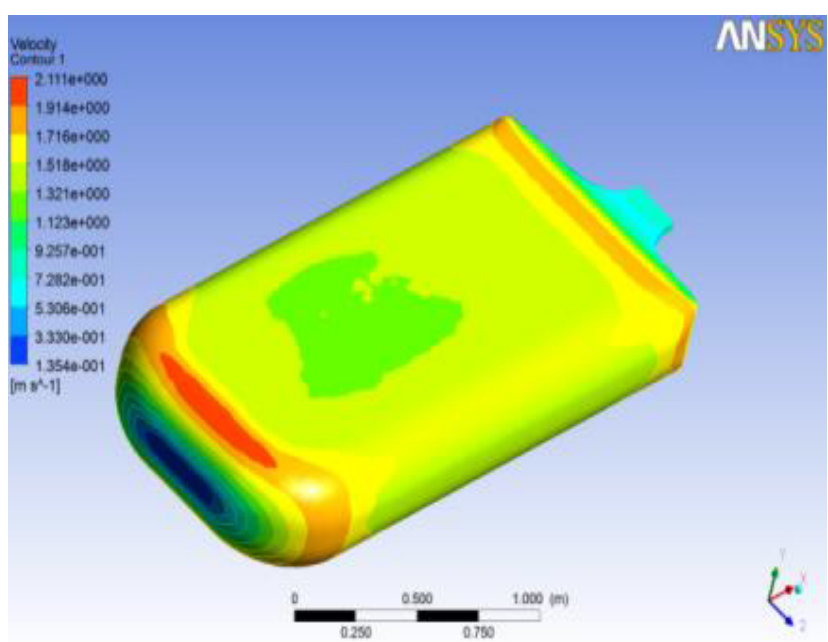

Figure 8. Pressure distribution at $3 \mathrm{kn}$

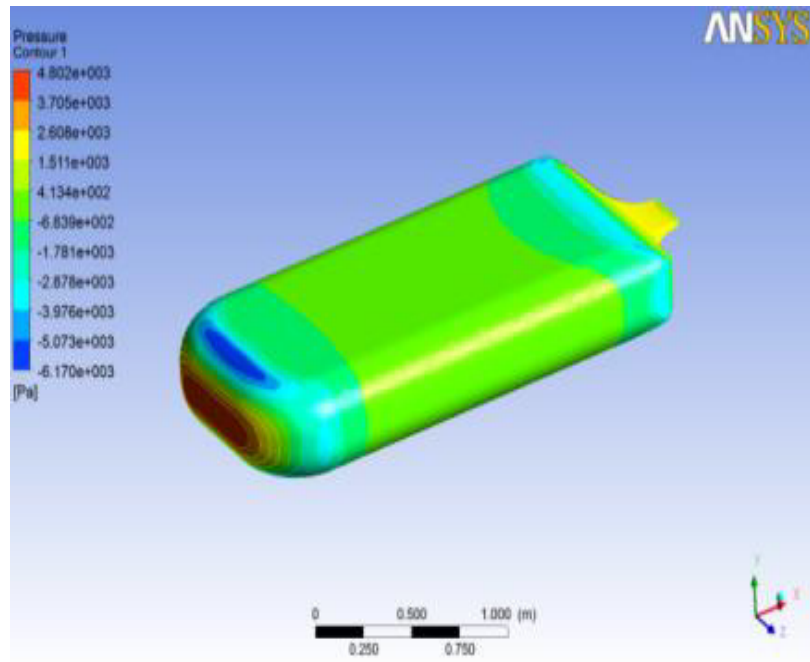

Figure 9. Velocity distribution at $6 \mathrm{kn}$

Figure 6. Pressure distribution at $1 \mathrm{kn}$ 


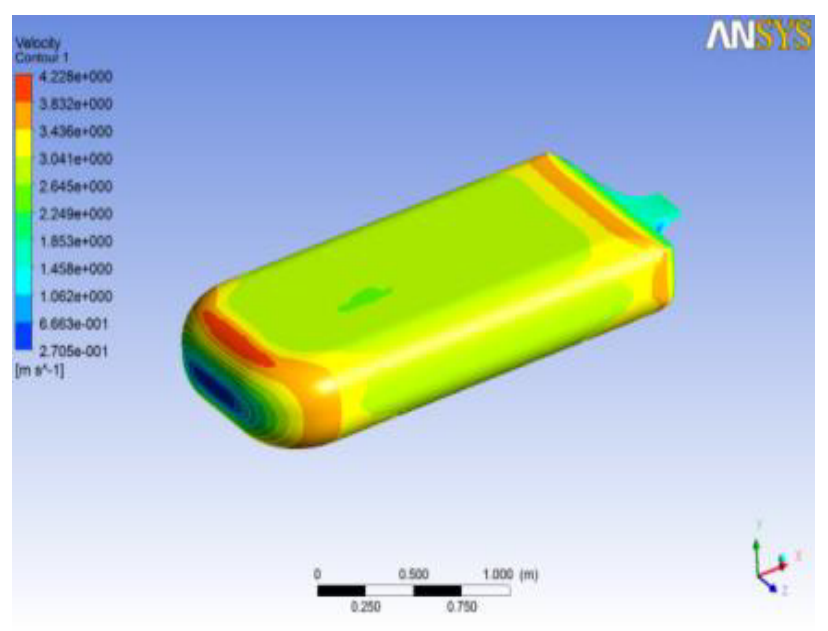

Figure 10. Pressure distribution at $6 \mathrm{kn}$

Table 3 Result of drag force with ARV CFD model at different speeds

\begin{tabular}{|c|c|c|c|}
\hline Speed & Drag Force & Speed & Drag Force \\
\hline 6 knots & $430[\mathrm{~N}]$ & 3 knots & $114[\mathrm{~N}]$ \\
\hline 5 knots & $303[\mathrm{~N}]$ & $2 \mathrm{knots}$ & $53[\mathrm{~N}]$ \\
\hline 4 knots & $197[\mathrm{~N}]$ & 1 knots & $15[\mathrm{~N}]$ \\
\hline
\end{tabular}

\section{Conclusion}

A CFD procedure has been conducted on the proposed ARV design. The drag estimation was conducted first using CFD analysis with a mesh convergence study performed to validate the CFD analysis. The hydrodynamic forces on the ARV body were obtained from the CFD motion analysis. The CFD modeling approach was then compared with towed body which was compared against the experimental data. Based on the validation studies, the CFD results have been shown to be reliable and the CFD analysis techniques employed are shown to be suitable for initial concept analysis of underwater fairing.

\section{References}

1. A. Budiyono, Advances in unmanned underwater vehicle technologies. Modeling, control and guidance perspectives. Indian Journal of Marine Sciences, 38(3), pp.282-295 (2009).

2. J. Yuh, G. Marani, D.R. Blidberg. Applications of marine robotic vehicles, Intell. Serv. Robotics 4(4), pp.221-231 (2011).

3. S. Q. Yang, H. L. Yang and M. W. Cao. Reliabilitybased Design for Pressure Shell of Submarine Pipeline Inspection AUV with 1,500 Meters Depth, Proceedings of the MSMD 2015, Changsha, 12-13 December, pp320-326 (2015).

4. A. Budiyono. Advances in unmanned underwater vehicle technologies: Modeling, control and guidance perspectives. Indian Journal of Marine Sciences, 38(3), pp.282-295 (2009).

5. T.H. Joung, K. Sammut, F. He. Shape optimization of an autonomous underwater vehicle with a ducted propeller using computational fluid dynamics analysis. International Journal of Naval Architecture \& Ocean Engineering, 4, pp.44-56 (2012).

6. X. Yu, Y. Su. Hydrodynamic performance calculation on mini-automatic underwater vehicle. Information and Automation (ICIA), 2010 IEEE International Conference, Harbin, 20-23 June, pp.1319-1324 (2010).

7. J.G. Bellingham, Y. Zhang, J.E. Kerwin. Efficient propulsion for the Tethys long-range autonomous underwater vehicle. Autonomous Underwater Vehicles (AUV), 2010 IEEE/OES, pp.1-7 (2010).

8. A.B. Phillips, R.T. Stephen and F. Maaten. Comparisons of CFD simulations and in-service data for the self propelled performance of an autonomous underwater vehicle. 27th Symposium on Naval Hydrodynamics, Seoul, Korea, 05-10 October 2008, pp.15 (2008).

9. D. F. Myring. A theoretical study of body drag in subcritical axisymmetric flow, Aeronaut. Q., vol. 27, no. 3, pp. 186-194 (1976).

10. Y. Nishi, M. Kashiwagi, and W. Koterayama,. Resistance and Propulsion Performance of an Underwater Vehicle Estimated by a CFD Method and Experiment, ISOPE '07, Lisbon, Spain, 1-6 July, pp.2045-2052 (2007). 